\title{
Characterization of microRNA expression in bovine adipose tissues: a potential regulatory mechanism of subcutaneous adipose tissue development
}

\author{
Weiwu Jin¹, Michael V Dodson², Stephen S Moore1, John A Basarab³ and Le Luo Guan*1
}

\begin{abstract}
Background: MicroRNAs (miRNAs), a family of small non-coding RNA molecules, appear to regulate animal lipid metabolism and preadipocyte conversion to form lipid-assimilating adipocytes (i.e. adipogenesis). However, no miRNA to date has been reported to modulate adipogenesis and lipid deposition in beef cattle.

Results: The expression patterns of 89 miRNAs including four bovine specific miRNAs in subcutaneous adipose tissues from three groups of crossbred steers differing in backfat thickness were compared using qRT-PCR analysis. Eighty-six miRNAs were detectable in all samples, with 42 miRNAs differing among crossbreds $(P<0.05)$ and 15 miRNAs differentially expressed between tissues with high and low backfat thickness $(P<0.05)$. The expression levels of 18 miRNAs were correlated with backfat thickness $(P<0.05)$. The miRNA most differentially expressed and the most strongly associated with backfat thickness was miR-378, with a 1.99-fold increase in high backfat thickness tissues $(r=$ 0.72).

Conclusions: MiRNA expression patterns differed significantly in response to host genetic components. Approximately $20 \%$ of the miRNAs in this study were identified as being correlated with backfat thickness. This result suggests that miRNAs may play a regulatory role in white adipose tissue development in beef animals.
\end{abstract}

\section{Background}

MicroRNAs (miRNAs) are small regulatory molecules (18 25 nucleotides) that are important in many biological processes including development, differentiation, apoptosis, and metabolism [1-5]. The role of miRNAs in lipid metabolism was first reported in Drosophila, where the deletion of miR-14 increased the levels of triacylglycerol and diacylglycerol [6]. Thereafter, several miRNAs were shown to promote preadipocyte differentiation in the human and mouse [7-9]. Analysis of the 3' UTR from 395 ESTs expressed in 3T3-L1 preadipocytes during conversion into lipid-assimilating adipocytes showed that $>70 \%$ of the differentially expressed genes may be potentially regulated by miRNAs [10]. A recent study on the expression of 155 miRNAs in human omental and subcutaneous adipose tissues found that the expression of miRNAs was adipose depot specific and that some miRNAs

* Correspondence: Iguan@ualberta.ca

${ }^{1}$ Department of Agricultural, Food and Nutritional Science, University of Alberta, Edmonton, AB, T6G2P5, Canada

Full list of author information is available at the end of the article were correlated with the morphology of adipose tissue and adipocyte size [11]. Although the roles of some miRNAs in lipid metabolism [6] and adipocyte formation [710] have been demonstrated, no miRNA has been reported to regulate lipid metabolism in beef cattle. Lipid deposition, especially in subcutaneous adipose tissues, is directly associated with the yield and the quality of meat [12]. We hypothesized that microRNAs play a role in the regulation of adipogenesis in bovine adipose tissue and the variation of expression of miRNAs is associated with differences in backfat thickness. To gain insight into the association between subcutaneous adipose tissue thickness and miRNA expression, the expression profiles of 89 miRNAs were determined in beef cattle subcutaneous adipose tissues of crossbred steers with different backfat thickness.

\section{Results and Discussion}

The aim of this study was to identify miRNAs which may play regulatory roles in adipose tissue development in beef cattle. 
The expression of miRNAs differed significantly in response to host genetic components

Analysis of the expression of 89 miRNAs including four bovine specific miRNAs (Additional file 1) in beef cattle back subcutaneous adipose tissues confirmed the expression of 86 miRNAs. The animals were divided into 6 groups, according to breed and level of backfat thickness. Hierarchical clustering of the detectable miRNAs showed the expression of miRNAs was more similar in animals of the same breed composition than animals with the same level of backfat thickness (Figure 1). We also compared miRNA expression among three crossbred groups and found the expression of 42 miRNAs to be significantly different among these groups. These results suggest that the expression of miRNAs may differ with regard to host genetic components. CHAR animals have miRNA expression patterns more similar with those of HEAN animals (Figure 1), with 17 significantly different miRNAs. Twenty-nine and 27 miRNAs were found to be significantly different between CHAM and CHAR crossbreds, and between CHAM and HEAN crossbreds, respectively. However, the backfat thickness of CHAR animals was significantly different than that of HEAN animals and was similar with that of CHAM animals (Figure 2).

\section{Differentially expressed miRNAs in adipose tissues from animals with different backfat thickness}

Fifteen differentially expressed (DE) miRNAs were identified between tissues from high and low backfat thickness (Figure 1, Table 1). Of these 15 DE miRNAs, seven (miR$378,-143,-760,-98,-196 \mathrm{a},-196 \mathrm{~b}$ and -107$)$ were identified as highly expressed in the tissue ( $>1.34$-fold) from high backfat thickness animals, whereas the remaining eight (miR-93, -151-5p, -214, -151-3p, -199a-3p, -191, 142-5p and -186) were expressed at a higher level in tissue from the low backfat thickness animals (Table 1). The most differentially expressed miRNA was miR-378 with a 1.99-fold increase in high backfat thickness tissues, suggesting that this miRNA may have a role in adipogenesis and/or lipid deposition in bovine backfat tissues.

In mouse the miR-17-92 cluster has been reported to promote preadipocyte differentiation [8]; and the miR17-92 cluster with other two cluster paralogs (miR-106a363 and miR-106b-25) were found to promote cell proliferation [13]. To test whether the miRNAs of the miR-1792 family are expressed higher in tissues with high backfat thickness, expression patterns of all members omitting miR-20b (Additional file 1, 2) were profiled. Unexpectedly, no DE miRNA from three clusters were identified, with the exception of miR-93 which was found 1.62-fold down-regulated in tissues from high backfat thickness animals compared to those from low backfat thickness animals $(\mathrm{P}>0.05)$. Our undetected expression difference of the miR-17-92 cluster from this study confirmed the previous observation that bovine adipogenesis had different molecular mechanisms than those in mice based on the study of gene expression patterns [14]. In addition, the miR-17-92 cluster was found to be up-regulated during the early clonal expansion stage of adipogenesis [8] and was not abundantly expressed in differentiated adipocytes [9]. Since backfat tissues are mixture of preadipocytes, adipocytes and other cells, the stage of adipogenesis could be very different from the pure adipocyte cultures. Further study to verify the expression of miR-17-92 using a bovine adipocyte cell line will provide a better understanding of this miRNA cluster in bovine adipogenesis. In addition, miR-143 was 1.66 -fold up-regulated in the tissues from high backfat animals, It has been reported that miR-143 was up-regulated during preadipocyte differentiation in human and mouse $[7,11,15]$, suggesting that this miRNA may promote bovine adipogenesis that may accelerate adipose tissue development.

\section{Backfat thickness associated miRNAs}

To confirm the above correlation between miRNA expression levels and backfat thickness, we further investigated the relationship between the expression of miRNAs and backfat thickness using Pearson correlation and simple linear regression analysis. Eighteen miRNAs, representing $20 \%$ of all analyzed miRNAs (Table 2), were identified as being significantly associated with backfat thickness in beef animals. Among the backfat thicknessassociated miRNAs, eight were DE miRNAs identified above, including two up-regulated miRNAs (miR-378, 196a) and six down-regulated miRNAs (miR-151-5p, 151-3p, -214, -186, -142-5p and -93) (Table 1). The most differentially expressed miRNA (miR-378) was shown to have the strongest correlation with backfat thickness ( $\mathrm{r}=$ 0.72 ). Four bovine specific miRNAs which were identified from our previous study [16] were also confirmed to be expressed in all the analyzed samples. Among them, one miRNA (bta-miR-3432) was significantly correlated to backfat thickness $(r=0.48)$, suggesting that this bovine specific miRNA may play a role in the adipogenesis of adipose tissues in cattle. The mechanism of how this miRNA influences bovine adipogenesis can be elucidated by studying the expression of this miRNA in bovine adipocyte cells and to identify its target genes.

Most of backfat thickness associated miRNAs in this study were found to be negatively correlated to backfat depth (Table 2). For example, the miR-106b-25 cluster, a miRNA cluster paralog of miR-17-92, was moderately negatively correlated with backfat thickness. These data suggest that miRNA may regulate white adipose tissue development, and that the difference in thickness of subcutaneous tissues may be one target. More research is 


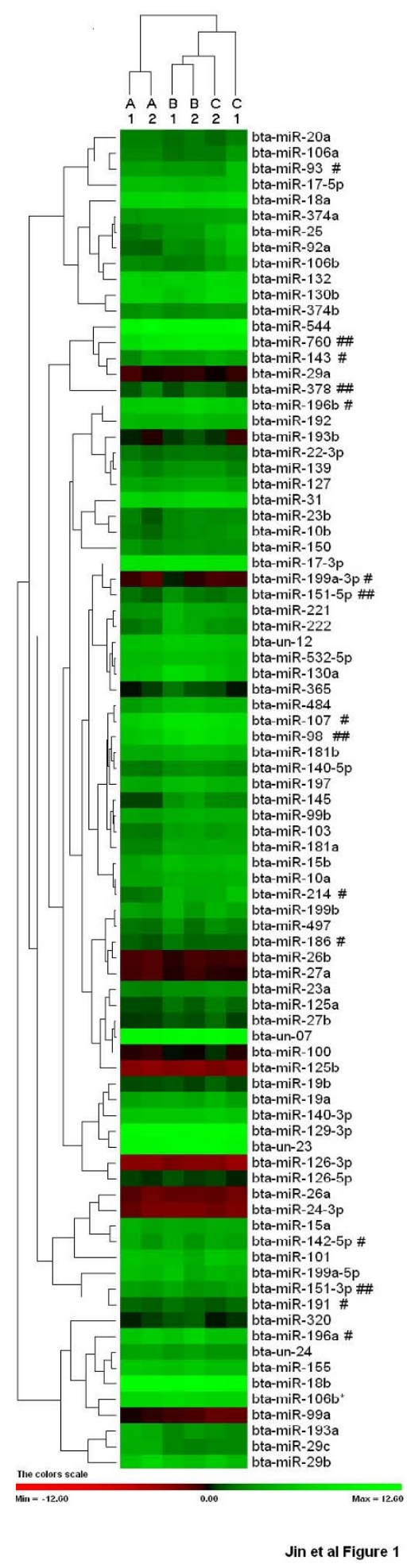

Figure 1 Expression pattern of miRNAs in back subcutaneous adipose tissues. Heatmap is constructed based on the mean expression levels of miRNAs in each group $(n=4)$. As shown by the scale bar, increasing green and red signal intensities indicate miRNA with lower or higher expression level, respectively. A1, CHAM with high backfat; A2, CHAM with low backfat; B1, CHAR with high backfat; B2, CHAR with low backfat; C1, HEAN with high backfat; C2, HEAN with low backfat. '\#' and '\#\#' indicate that miRNA expression was significant or very significant difference between tissues with high and low backfat thickness, respectively. 


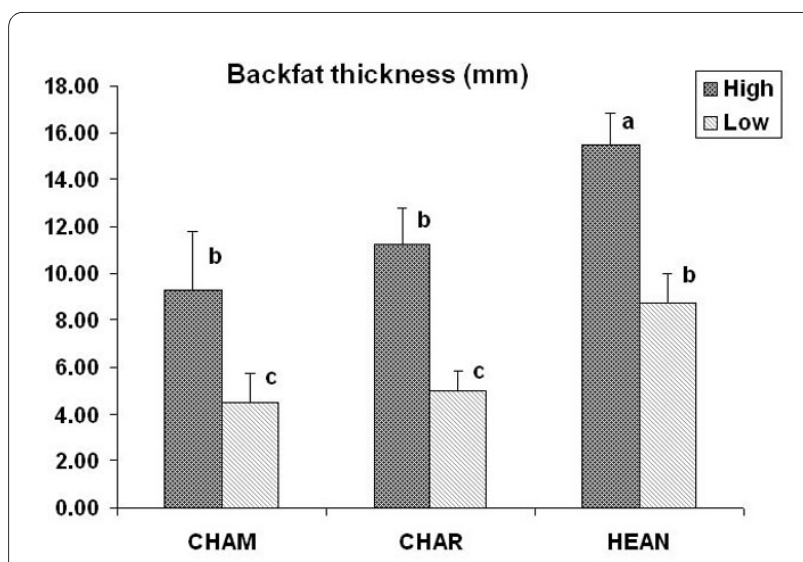

Figure $\mathbf{2}$ Comparisons of backfat thickness between groups. Oneway ANOVA was performed. Groups with the same letter are not significantly different.

needed to verify this and to provide intracellular/gene mechanisms.

\section{Potential role of miR-378 in bovine adipogenesis}

Approximately two thirds of the miRNAs that were correlated with backfat thickness in this study were identified in introns or exons of genes (Table 2). Intronic miRNAs may be coordinately expressed with their targeted gene mRNAs [17-19] and may act synergistically with the targeted genes [20]. MiR-378, the most DE and the strongest associated with backfat thickness, was found to be located in intron 1 of peroxisome proliferator-activated receptor gamma coactivator 1 beta (PGC-1 $\beta$ ) (Table 2). Since PGC-1 $\beta$ has been shown to be induced during brown adipocyte differentiation [21] and to increase lipogenesis and lipoprotein transport in the liver [22,23], we suggest that PGC-1 $\beta$ may play a role in white adipose tissue development. Furthermore, a search of Microcosm Targets V5 http://www.ebi.ac.uk/enright-srv/microcosm/ htdocs/targets/v5/ with miR-378 identified 816 potential target genes. One interesting target gene is mitogen-activated protein kinase 1 (MAPK1). MAPK1 can mediate phosphorylation of the dominant adipogenic transcription factor peroxisome proliferator-activated receptor $\gamma$ (PPAR $\gamma)$ and reduce its transcriptional activity [24-26]. PPAR $\gamma$ is up regulated in bovine preadipocyte adipogenesis, [27,28] and the knock-down of PPAR $\gamma$ markedly inhibited preadipocyte-to adipocyte conversion in 3T3L1 cells [29]. Therefore, we speculate that miR-378 may promote bovine adipogenesis in white adipose tissue through targeting MAPK1 and PPAR $\gamma$ (Figure 3). However, this regulatory mechanism may be one of the many other functions this miRNA may have. Since miRNA-378 can also target many other genes, future studies on the relationships of miRNA target genes and how the network of these target genes could impact on white adipose tissue development will supply a comprehensive view of the molecular mechanisms of fat formation, a very complicated process in beef cattle.

Table 1: Differentially expressed miRNAs between high and low backfat thickness fat tissues.

\begin{tabular}{|c|c|c|c|c|}
\hline bta-miRNA & $\begin{array}{l}\text { High }(n=12) \\
\text { Mean } \pm \text { SD }\end{array}$ & $\begin{array}{l}\text { Low }(n=12) \\
\text { Mean } \pm \text { SD }\end{array}$ & -Fold change & p-value \\
\hline miR-378 & $0.84 \pm 0.38$ & $1.83 \pm 0.53$ & 1.99 & $<0.0001$ \\
\hline miR-760 & $7.95 \pm 0.82$ & $8.65 \pm 0.43$ & 1.62 & 0.0005 \\
\hline miR-151-3p & $3.51 \pm 0.44$ & $3.02 \pm 0.15$ & -1.40 & 0.0022 \\
\hline miR-151-5p & $2.19 \pm 0.73$ & $1.59 \pm 0.44$ & -1.52 & 0.0050 \\
\hline miR-98 & $6.19 \pm 0.95$ & $6.85 \pm 1.05$ & 1.58 & 0.0097 \\
\hline miR-196a & $5.10 \pm 0.35$ & $5.68 \pm 0.81$ & 1.49 & 0.0101 \\
\hline miR-214 & $3.79 \pm 1.64$ & $3.21 \pm 1.03$ & -1.50 & 0.0101 \\
\hline miR-93 & $3.78 \pm 1.13$ & $3.08 \pm 0.35$ & -1.62 & 0.0106 \\
\hline miR-196b & $5.56 \pm 0.50$ & $6.09 \pm 0.53$ & 1.44 & 0.0148 \\
\hline miR-107 & $6.83 \pm 0.97$ & $7.25 \pm 0.81$ & 1.34 & 0.0173 \\
\hline miR-186 & $1.55 \pm 0.41$ & $1.21 \pm 0.34$ & -1.27 & 0.0178 \\
\hline miR-199a-3p & $-0.29 \pm 0.49$ & $-0.69 \pm 0.51$ & -1.32 & 0.0198 \\
\hline miR-191 & $1.57 \pm 0.46$ & $1.17 \pm 0.28$ & -1.32 & 0.0202 \\
\hline miR-142-5p & $3.69 \pm 0.66$ & $3.14 \pm 0.57$ & -1.47 & 0.0266 \\
\hline miR-143 & $3.03 \pm 0.71$ & $3.76 \pm 0.85$ & 1.66 & 0.0268 \\
\hline
\end{tabular}

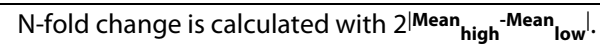


Table 2: Backfat thickness associated miRNAs.

\begin{tabular}{|c|c|c|c|c|}
\hline miRNA & $\begin{array}{l}\text { Mean } \pm \text { SD } \\
(n=24)\end{array}$ & r (p-value) & miRNA location 1 & Gene description2 \\
\hline miR-196a & $5.39 \pm 0.68$ & $0.65(0.0006)$ & Intergenic & \\
\hline bta-miR-3432 & $3.22 \pm 0.47$ & $0.48(0.0177)$ & - (Intergenic) & \\
\hline $\operatorname{miR}-92 a$ & $2.75 \pm 1.65$ & $-0.58(0.0028)$ & Intronic (Intergenic) & chromosome 13 open reading frame 25 (-) \\
\hline miR-151-3p & $3.27 \pm 0.41$ & $-0.53(0.0076)$ & Intronic & protein tyrosine kinase 2 \\
\hline miR-151-5p & $1.89 \pm 0.67$ & $-0.49(0.0154)$ & Intronic & protein tyrosine kinase 2 \\
\hline miR-214 & $3.50 \pm 1.37$ & $-0.52(0.0094)$ & Intronic & dynamin 3 (similar to dynamin 3 ) \\
\hline miR-106b & $2.68 \pm 0.86$ & $-0.49(0.0151)$ & Intronic & mini-chromosome maintenance protein 7 \\
\hline miR-93 & $3.43 \pm 0.89$ & $-0.43(0.0343)$ & Intronic & mini-chromosome maintenance protein 7 \\
\hline miR-199b & $3.59 \pm 0.86$ & $-0.48(0.0188)$ & Intronic & dynamin 1 \\
\hline miR-10b & $2.36 \pm 0.98$ & $-0.45(0.0282)$ & Intergenic & \\
\hline miR-186 & $1.38 \pm 0.41$ & $-0.44(0.0319)$ & Intronic & $\begin{array}{l}\text { zinc finger, RAN-binding domain containing } \\
2 \text { (zinc finger protein } 265 \text { ) }\end{array}$ \\
\hline miR-23b & $2.24 \pm 0.84$ & $-0.43(0.0386)$ & Intronic (Intergenic) & chromosome 9 open reading frame $3(-)$ \\
\hline miR-132 & $6.25 \pm 0.63$ & $-0.42(0.0392)$ & Intergenic & \\
\hline miR-101 & $4.82 \pm 0.57$ & $-0.42(0.0412)$ & Intronic & RNA terminal phosphate cyclase-like \\
\hline miR-27a & $-0.47 \pm 0.69$ & $-0.41(0.0456)$ & Intergenic & \\
\hline miR-142-5p & $3.41 \pm 0.66$ & $-0.41(0.0487)$ & Intergenic & \\
\hline
\end{tabular}

${ }^{1}$ miRNA location is listed. If the human/cattle positions are different, the cattle location is presented in parentheses. Data are from miRBase.

${ }^{2} \mathrm{Gene}$ is listed. If the human/cattle genes are different, the cattle gene is presented in parentheses. Data are from miRBase, NCBI and Ensembl.

'-' means not find so far. *This gene in bovine is named IPI00715774.3 (Ensembl) and shares homology to human PGC-1 $\beta$ (Blastp).

\section{Conclusions}

We assessed the miRNA expression patterns in the subcutaneous (backfat) adipose depot of crossbred beef cattle and found that the expression patterns of miRNA were different depending on host genetic components. In total, 86 miRNA were detected, and 18 miRNAs were identified as being associated with backfat thickness, suggesting that miRNAs may play an important role in white adipose tissue development in beef cattle. MiRNA-378 was found to be the most DE and strongly associated miRNA with

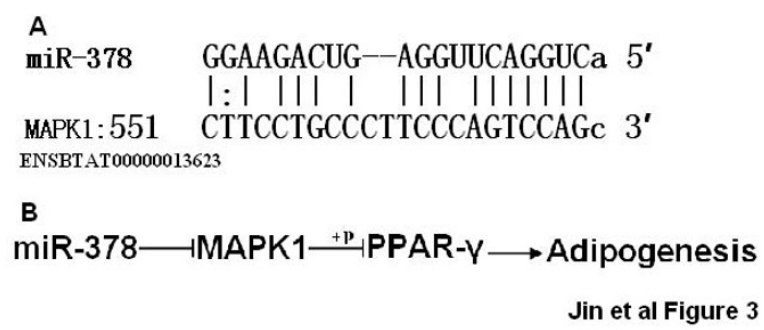

Figure 3 Hypothesis of miR-378 in bovine adipogenesis. A. Potential target of miR-378 was predicted in the 3' UTR of MAPK1 (Ensembl ID and position are listed). B. Regulation pathway of miR-378 in adipogenesis. backfat thickness. This miRNA has a potential function of regulating bovine adipogenesis by targeting MAPK1 and of PPAR $\gamma$. Future work to study miRNA expression of adipose collected from different growth stage, among different adipose tissue depots, or from key individual cell populations derived from adipose tissue proper, is necessary to elucidate the functions of miRNA in bovine lipid metabolism. These will prove useful knowledge to reduce the development of inefficient adipose tissue depots (subcutaneous depot), while enhancing the formation of desired depots (intramuscular depot) in beef cattle. Such knowledge may also apply to humans, especially in terms of regulation of metabolic syndrome, diabetes, or obesity.

\section{Methods}

Animals and total RNA extraction

All animals in this study were maintained at the Lacombe Research Centre, Lacombe, Alberta, Canada, and were cared for according to the guidelines of the Canadian Council on Animal Care (CCAC 1993). The animal use authorization form was approved by the Animal Care Committee at the Lacombe Research Centre. Hereford $x$ Aberdeen Angus (HEAN) $(\mathrm{n}=22)$, Charolais $\times$ Red 
Angus (CHAR) $(\mathrm{n}=13)$, and Charolais $\times$ Maine Anjou (CHAM) $(n=9)$ crossbred steers were fed on a finishing diet composed of approximately $73.3 \%$ barley grain, $22.0 \%$ barley silage, $1.6 \%$ molasses, and $3.1 \%$ feedlot supplement [32\% CP beef supplement containing Rumensin (400 mg kg-1) fed ad libitum] and slaughtered at 14-17 mo. Details of feeding and management were described by Basarab et al. [30]. Carcass traits including backfat thickness, estimated cutability, marbling score, and other information were recorded. The subcutaneous adipose tissues above the 10-11th rib were collected immediately after the animals were harvested at the Lacombe
Research Centre abattoir, frozen in liquid nitrogen and stored at $-80^{\circ} \mathrm{C}$ until further analysis. Samples were ranked by backfat thickness at the 12-13th rib (also know as grade fat). Adipose tissues of the top four and the bottom four in each crossbred were selected and divided into high and low backfat thickness groups (Table 3).

Total RNA was extracted with mirVana miRNA Isolation Kit (Ambion Inc, Austin, TX, USA) according to the manufacturer's instruction. The quality and concentration of total RNA were determined by routine agarose gel analysis and spectrophotometer ND-1000 (Thermo Scientific, Waltham, MA, US), respectively.

Table 3: Carcass characteristics of animals used in this study.

\begin{tabular}{|c|c|c|c|c|c|c|c|c|}
\hline \multirow[t]{2}{*}{ Breed } & \multirow[t]{2}{*}{ Animal ID } & \multicolumn{4}{|c|}{ Fat $(\mathrm{mm})$} & \multirow[t]{2}{*}{ Cutability (\%) } & \multirow[t]{2}{*}{ Age (Day) } & \multirow{2}{*}{$\begin{array}{c}\text { Slaughter } \\
\text { Weight (kg) }\end{array}$} \\
\hline & & top & mid & bottom & 12-13th rib & & & \\
\hline \multirow{4}{*}{$\begin{array}{c}\text { CHAR }^{\text {a }} \\
\text { (Low backfat) }\end{array}$} & 301 & 8 & 6 & 5 & 4 & 64 & 465 & 571.5 \\
\hline & 107 & 5 & 5 & 5 & 5 & 64 & 459 & 651.5 \\
\hline & 403 & 10 & 6 & 6 & 5 & 61 & 488 & 456.0 \\
\hline & 309 & 10 & 6 & 7 & 6 & 62 & 486 & 557.5 \\
\hline \multirow{4}{*}{$\begin{array}{c}\text { CHAR } \\
\text { (High backfat) }\end{array}$} & 106 & 13 & 11 & 11 & 10 & 59 & 458 & 627.5 \\
\hline & 410 & 15 & 11 & 11 & 10 & 56 & 485 & 504.5 \\
\hline & 209 & 14 & 12 & 12 & 12 & 55 & 449 & 542.5 \\
\hline & 210 & 16 & 14 & 14 & 13 & 56 & 476 & 558.0 \\
\hline \multirow{4}{*}{$\begin{array}{c}\text { CHMA }^{b} \\
\text { (Low backfat) }\end{array}$} & 111 & 6 & 6 & 4 & 3 & 65 & 434 & 578.5 \\
\hline & 303 & 9 & 5 & 5 & 4 & 62 & 485 & 563.5 \\
\hline & 407 & 6 & 5 & 5 & 5 & 62 & 476 & 548.0 \\
\hline & 101 & 6 & 4 & 6 & 6 & 62 & 471 & 687.5 \\
\hline \multirow{4}{*}{$\begin{array}{c}\text { CHMA } \\
\text { (High backfat) }\end{array}$} & 201 & 9 & 8 & 7 & 6 & 61 & 467 & 581.5 \\
\hline & 103 & 10 & 10 & 8 & 8 & 61 & 465 & 634.0 \\
\hline & 113 & 9 & 9 & 8 & 8 & 61 & 470 & 670.5 \\
\hline & 112 & 13 & 13 & 13 & 13 & 58 & 462 & 688.0 \\
\hline \multirow{4}{*}{$\begin{array}{c}\text { HEANc } \\
\text { (Low backfat) }\end{array}$} & 401 & 13 & 12 & 8 & 7 & 59 & 501 & 540.5 \\
\hline & 207 & 10 & 9 & 9 & 9 & 58 & 458 & 573.5 \\
\hline & 205 & 14 & 11 & 10 & 10 & 58 & 463 & 590.0 \\
\hline & 310 & 13 & 9 & 9 & 9 & 60 & 482 & 576.5 \\
\hline \multirow{4}{*}{$\begin{array}{c}\text { HEAN } \\
\text { (High backfat) }\end{array}$} & 405 & 18 & 15 & 14 & 14 & 53 & 494 & 507.0 \\
\hline & 208 & 19 & 17 & 16 & 15 & 53 & 479 & 519.0 \\
\hline & 102 & 24 & 19 & 19 & 16 & 54 & 472 & 626.5 \\
\hline & 203 & 16 & 18 & 18 & 17 & 52 & 459 & 504.5 \\
\hline
\end{tabular}

aCHAR, Charolais $\times$ Red Angus; ${ }^{b}$ CHAM, Charolais $\times$ Maine Anjou; cHEAN, Hereford $\times$ Aberdeen Angus. 


\section{MicroRNA expression using qRT-PCR}

Expression of 89 miRNAs including four miRNA candidates was carried out with $\mathrm{TaqMan}^{\circ}$ miRNA assays according to the manufacturer's recommendation (Applied Biosystems, Foster City, CA, USA). Briefly, cDNA was reversely transcribed from 10 ng of total RNA using $5 \times$ specific miRNA RT primer, respectively. PCR products were amplified from cDNA samples using $20 \times$ TaqMan miRNA Assays. Fluorescence signal was detected on an ABI StepOnePlus Real-time PCR System detector (Applied Biosystems, Foster City, CA, USA). Triplicates or four replicates for each reaction were performed. Bovine miR-16b was used as an endogenous control to normalize the expression levels of the other miRNAs. Eighty-five miRNA assays were selected from mouse, rat or human. Most of them had $100 \%$ identity in sequence with bovine miRNAs but some had one or two nucleotides longer or shorter at the $3^{\prime}$ or $5^{\prime}$ end. Only miR-143 and miR-363 had one and two nucleotides difference at the 3 ' between bovine and human, respectively (Additional file 1). The other four assays for bovine specific miRNAs (bta-miR-2284w, bta-miR-3431, bta-miR2284x and bta-miR-3432, Additional file 1) were synthesized by Applied Biosystems (Foster City, CA, USA), and the target sequences were selected from our recently identified bovine miRNA candidates [16].

\section{Data analyses}

MiRNA expression $\left(\Delta \mathrm{C}_{\mathrm{T}}\right)$ was analyzed by 2-way ANOVA using GLM procedures of SAS 9.2 (2008) for the fixed effects of backfat, crossbred group, backfat $\times$ crossbred group. A P value $<0.05$ was considered to be statistically significant. miRNA expression between either two crossbred groups was contrasted using 'means breed/ Tukey LSD', and the P value was adjusted to 0.017 for statistical significance. The relationships between miRNA expression and backfat thickness were assessed by Pearson's correlation, and simple linear regression was done after the data of backfat thickness were transformed as $\log 2$ ratios. The correlation between either two miRNAs was also evaluated by Pearson's correlation. Hierarchical clustering of miRNA expression was performed using PermutMatrix software with Pearson distance for dissimilarity, average linkage for hierarchical clustering and multiple-fragment heuristic for seriation [31].

\section{Additional material}

Additional file $1 \mathrm{TaqMan}^{\circ}$ miRNA Assays.

Additional file 2 The genomic organization of the miR-17-92 family.

\section{Authors' contributions}

LLG and WJ designed the experiments; WJ performed QRT-PCR, data analysis, and original manuscript writing; MVD, SSM and JAB were involved in discus- sion and manuscript construction. WJ and LLG wrote the paper. All authors read and approved the final manuscript.

\section{Acknowledgements}

This study is supported by the Alberta Livestock Industry Development Fund (2007F044R). The authors thank Dr. M. Taniguchi for collecting samples, and Dr. U. Basu and Ms. Y. Meng for technical support.

\section{Author Details}

'Department of Agricultural, Food and Nutritional Science, University of Alberta, Edmonton, AB, T6G2P5, Canada, 2Department of Animal Sciences, Washington State University, PO Box 646310, Pullman, Washington, 99164 USA and ${ }^{3}$ Alberta Agriculture and Rural Development, Lacombe Research Centre, Lacombe, AB, T4L1W1, Canada

Received: 11 September 2009 Accepted: 27 April 2010

Published: 27 April 2010

\section{References}

1. Alvarez-Garcia I, Miska EA: MicroRNA functions in animal development and human disease. Development 2005, 132(21):4653-4662.

2. Lee CT, Risom T, Strauss WM: MicroRNAs in mammalian development. Birth Defects Res CEmbryo Today 2006, 78(2):129-139.

3. O'Rourke JR, Swanson MS, Harfe BD: MicroRNAs in mammalian development and tumorigenesis. Birth Defects Res CEmbryo Today 2006, 78(2):172-179.

4. Song L, Tuan RS: MicroRNAs and cell differentiation in mammalian development. Birth Defects Res CEmbryo Today 2006, 78(2):140-149.

5. Krutzfeldt J, Stoffel M: MicroRNAs: a new class of regulatory genes affecting metabolism. Cell Metab 2006, 4(1):9-12.

6. Xu P, Vernooy SY, Guo M, Hay BA: The Drosophila microRNA Mir-14 suppresses cell death and is required for normal fat metabolism. Curr Biol 2003, 13(9):790-795

7. Esau C, Kang X, Peralta E, Hanson E, Marcusson EG, Ravichandran LV, Sun Y, Koo S, Perera RJ, Jain R, et al:: MicroRNA-143 Regulates Adipocyte Differentiation. J Biol Chem 2004, 279(50):52361-52365.

8. Wang Q, Li YC, Wang J, Kong J, Qi Y, Quigg RJ, Li X: miR-17-92 cluster accelerates adipocyte differentiation by negatively regulating tumorsuppressor Rb2/p130. PNAS 2008, 105(8):2889-2894.

9. Xie H, Lim B, Lodish HF: MicroRNAs Induced During Adipogenesis that Accelerate Fat Cell Development Are Downregulated in Obesity. Diabetes 2009, 58(5):1050-1057.

10. Hackl H, Burkard TR, Sturn A, Rubio R, Schleiffer A, Tian S, Quackenbush J, Eisenhaber F, Trajanoski Z: Molecular processes during fat cell development revealed by gene expression profiling and functional annotation. Genome Biol 2005, 6(13):R108

11. Kloting N, Berthold S, Kovacs P, Schon MR, Fasshauer M, Ruschke K, Stumvoll M, Bluher M: MicroRNA expression in human omental and subcutaneous adipose tissue. PLoS One 2009, 4(3):e4699.

12. Powell WE, Huffman DL: Predicting Chemical Composition of Beef Carcasses from Easily Obtainable Carcass Variables. J Anim Sci 1973, 36(6):1069-1076.

13. Mendell JT: miRiad roles for the miR-17-92 cluster in development and disease. Cell 2008, 133(2):217-222

14. Taniguchi M, Guan LL, Zhang B, Dodson MV, Okine E, Moore SS: Gene expression patterns of bovine perimuscular preadipocytes during adipogenesis. Biochem Biophys Res Commun 2008, 366(2):346-351.

15. Kajimoto K, Naraba H, Iwai N: MicroRNA and 3T3-L1 pre-adipocyte differentiation. RNA 2006, 12(9):1626-1632.

16. Jin W, Grant J, Stothard P, Moore S, Guan L: Characterization of bovine miRNAs by sequencing and bioinformatics analysis. BMC Molecular Biology 2009, 10(1):90.

17. Baskerville S, Bartel DP: Microarray profiling of microRNAs reveals frequent coexpression with neighboring miRNAs and host genes. RNA 2005, 11(3):241-247.

18. Ying SY, Lin SL: Current perspectives in intronic micro RNAs (miRNAs). J Biomed Sci 2006, 13(1):5-15.

19. Lin SL, Miller JD, Ying SY: Intronic MicroRNA (miRNA). J Biomed Biotechnol 2006, 2006(4):26818.

20. Wilfred $B R$, Wang $W X$, Nelson PT: Energizing miRNA research: a review of the role of miRNAs in lipid metabolism, with a prediction that miR-103/ 
107 regulates human metabolic pathways. Mol Genet Metab 2007, 91(3):209-217.

21. Lin J, Puigserver P, Donovan J, Tarr P, Spiegelman BM: Peroxisome Proliferator-activated Receptor gamma Coactivator 1 beta (PGC-1 beta), A Novel PGC-1-related Transcription Coactivator Associated with Host Cell Factor. J Biol Chem 2002, 277(3):1645-1648.

22. Lin J, Yang R, Tarr PT, Wu PH, Handschin C, Li S, Yang W, Pei L, Uldry M, Tontonoz $P$, et al: Hyperlipidemic effects of dietary saturated fats mediated through PGC-1 beta coactivation of SREBP. Cell 2005, 120(2):261-273.

23. Wolfrum C, Stoffel M: Coactivation of Foxa2 through Pgc- 1 beta promotes liver fatty acid oxidation and triglyceride/VLDL secretion. Cell Metab 2006, 3(2):99-110.

24. Hu E, Kim JB, Sarraf P, Bruce MN, Spiegelman N: Inhibition of Adipogenesis Through MAP Kinase-Mediated Phosphorylation of PPARgamma. Science 1996, 274(5295):2100-2103.

25. Adams M, Reginato MJ, Shao D, Lazar MA, Chatterjee VK: Transcriptional Activation by Peroxisome Proliferator-activated Receptor gamma Is Inhibited by Phosphorylation at a Consensus Mitogen-activated Protein Kinase Site. J Bio/ Chem 1997, 272(8):5128-5132.

26. Camp HS, Tafuri SR: Regulation of Peroxisome Proliferator-activated Receptor gamma Activity by Mitogen-activated Protein Kinase. J Biol Chem 1997, 272(16):10811-10816.

27. Rangwala SM, Lazar MA: Transcriptional control of adipogenesis. Annu Rev Nutr 2000, 20:535-559.

28. Rosen ED, Walkey CJ, Puigserver P, Spiegelman BM: Transcriptional regulation of adipogenesis. Genes \& Dev 2000, 14(11):1293-1307.

29. Katayama K, Wada K, Miyoshi H, Ohashi K, Tachibana M, Furuki R, Mizuguchi H, Hayakawa T, Nakajima A, Kadowaki T, et al:: RNA interfering approach for clarifying the PPARgamma pathway using lentiviral vector expressing short hairpin RNA. FEBS Lett 2004, 560(1-3):178-182.

30. Basarab JA, McCartney D, Okine EK, Baron VS: Relationships between progeny residual feed intake and dam productivity traits. Can J Anim Sci 2007, 87(4):489-502.

31. Caraux G, Pinloche S: PermutMatrix: a graphical environment to arrange gene expression profiles in optimal linear order. Bioinformatics 2005, 21(7):1280-1281.

doi: 10.1186/1471-2199-11-29

Cite this article as: Jin et al., Characterization of microRNA expression in bovine adipose tissues: a potential regulatory mechanism of subcutaneous adipose tissue development BMC Molecular Biology 2010, 11:29

Submit your next manuscript to BioMed Centra and take full advantage of:

- Convenient online submission

- Thorough peer review

- No space constraints or color figure charges

- Immediate publication on acceptance

- Inclusion in PubMed, CAS, Scopus and Google Scholar

- Research which is freely available for redistribution

Submit your manuscript at www.biomedcentral.com/submit
C Biomed Central 